Fernie Penning-van Beest · Joëlle Erkens

Karl-Uwe Petersen · Hans Rudolf Koelz · Ron Herings

\title{
Main comedications associated with major bleeding during anticoagulant therapy with coumarins
}

Received: 14 January 2005 / Accepted: 16 April 2005/Published online: 10 June 2005

(C) Springer-Verlag 2005

\begin{abstract}
Objective: To study the main comedications associated with major bleeding during anticoagulant therapy with coumarins in a non-selected population under everyday circumstances.

Methods: The study population for this retrospective cohort study included all new users of phenprocoumon or acenocoumarol aged 40-80 years, during the period 1992-2000 in the PHARMO Record Linkage System. All patients were followed until the last dispensing of phenprocoumon or acenocoumarol, the first bleeding complication requiring hospitalization, death, or the end of the study period. The number of days on coumarins alone and the number of days on coumarins in combination with several potentially interactive drugs during follow-up were determined for each patient.

Results: The inclusion criteria of this study were met by 19,935 new users of phenprocoumon or acenocoumarol. During follow-up, 552 patients were hospitalized for bleeding. Of all potentially interactive drugs started during anticoagulant therapy by at least 50 patients and with at least five bleedings, antibacterial drugs were associated with a four to seven times increased risk of bleeding. Among non-steroidal anti-inflammatory drugs, naproxen had the highest relative risk. Anti-
\end{abstract}

F. Penning-van Beest $(\bowtie) \cdot$ J. Erkens $\cdot$ R. Herings

PHARMO Institute, P. O. Box 85222, 3508 AE, Utrecht,

The Netherlands

E-mail: fernie.penning@pharmo.nl

Tel.: + 31-0-30-234-5162

Fax: + 31-0-30-234-5568

K.-U. Petersen

Rheinisch-Westfälische Technische Hochschule,

Aachen, Germany

H. R. Koelz

Division of Gastroenterology, Department of Medicine,

Triemli Hospital, Zurich, Switzerland

R. Herings

Department of Pharmaco-epidemiology and Pharmacotherapy,

Utrecht Institute for Pharmaceutical Sciences, Utrecht University, Utrecht, The Netherlands thrombotic salicylates and tramadol were associated with a three times increased risk of bleeding.

Conclusion: Antibacterial drugs, non-steroidal antiinflammatory drugs, antithrombotic salicylates and tramadol were the main potentially interactive drugs associated with major bleeding during anticoagulant therapy with coumarins under everyday circumstances.

\section{Introduction}

Major or life-threatening hemorrhage during anticoagulant therapy with coumarins occurs at an estimated rate of 1.1-3.6 per 100 patient-years [11, 15, 16]. Several treatment-specific and patient-specific risk factors for bleeding complications have been described [11]. Among others, the risk of bleeding is strongly associated with the intensity of anticoagulation. Consequently, comedications that enhance the anticoagulant effect of coumarin anticoagulants may be associated with an increased risk of bleeding. The critical period is the start of treatment with these comedications [18]. Comedications that interfere with hemostasis or have an ulcerogenic effect also increase the risk of bleeding during anticoagulant therapy with coumarins [13].

Most information on drug interactions with coumarin anticoagulants is based on case reports and smallscale experiments $[1,4,7,18]$. This is an unsatisfactory basis on which to make recommendations for anticoagulant therapy. Recently, some large-scale epidemiological studies on the association between potentially interacting drugs and overanticoagulation have been performed [17, 21-23]. We conducted a follow-up study in a large population-based cohort to investigate the main comedications associated with major bleeding during anticoagulant therapy with coumarins. The study was restricted to the start of treatment with potentially interactive drugs (PIDs) that might enhance the anticoagulant effect of coumarin anticoagulants or otherwise increase the risk of bleeding. 


\section{Methods}

\section{Setting}

Data were obtained from the PHARMO Record Linkage System, which includes among other databases the drug-dispensing records from community pharmacies and hospital discharge records of all 950,000 community-dwelling inhabitants of 40 demographically defined areas in The Netherlands. The computerized drug-dispensing histories contain data concerning the dispensed drug, type of prescriber, dispensing date, dispensed amount, prescribed dose regimens, and the legend duration of use (prescription length). All drugs are coded according to the Anatomical Therapeutic Chemical (ATC) Classification. The hospital records include detailed information concerning the primary and secondary diagnoses, procedures, and dates of hospital admission and discharge. All diagnoses are coded according to the International Classification of Diseases, Ninth Revision, Clinical Modification (ICD9-CM). For a detailed description of the database, we refer to previous work [5]. The database allows, without selection bias, identification of patients with certain drug prescriptions and hospital diagnoses.

\section{Study cohort}

The source population included all new users (incident users) of phenprocoumon or acenocoumarol in The Netherlands during the period 1992-2000. A patient was defined as a new user of phenprocoumon or acenocoumarol if none of these drugs had been dispensed for at least 1 year before the first phenprocoumon or acenocoumarol dispensing that appeared in the PHARMO database. All new users of phenprocoumon or acenocoumarol aged 40-80 years with a registration in the PHARMO database of at least 1 year were included in the study cohort.

\section{Identification of frequently used PIDs}

As we were interested in the main comedications associated with major bleeding during anticoagulant therapy, we first identified frequently used PIDs (i.e., used by at least 50 patients).

The following comedications were considered as PIDs: all drugs enhancing the anticoagulant effect or otherwise increasing the risk of bleeding, as listed in the Dutch standard on coumarin interactions, and all inhibitors of cytochrome $P_{450}$ (CYP) 1A2, 2C19 and $2 \mathrm{C} 9[3,8]$. As pantoprazole has recently been found to be a competitive inhibitor of both CYP2C9 and CYP2C19 [12], this proton pump inhibitor (PPI) was also included along with other commonly used PPIs. In total, 37 individual drugs and 9 drug groups were considered.
To study the incidence of PID use during anticoagulant therapy, treatment episodes with coumarins during the first year of follow-up were established for each patient [2]. In general, a treatment episode is defined as a period of time in which a continuous specific pharmacotherapeutic treatment takes place. To establish treatment episodes, the duration of use of each prescription was calculated by dividing the number of units dispensed by the number of units to be used per day, as defined in the pharmacies. If the gap between the end of one prescription and the start of the next prescription was less than half of the usual treatment duration, the treatment was considered to be uninterrupted. The treatment episode was measured as the time span between the starting date of the first prescription and the expiry date of the final prescription. The latter was set at half of the time period since the last dispensing date, assuming that patients may stop their medication prematurely. Patients may have had more than one treatment episode. For each PID and group using PIDs, the number of patients who had started using such a drug during a coumarin treatment episode was assessed. For those groups in which PID was used by at least 50 patients, the individual PIDs within this group were determined.

\section{Association with bleeding}

To study the association with bleeding for frequently used PIDs (i.e., used by at least 50 patients), all patients were followed from the first phenprocoumon or acenocoumarol dispensing in the PHARMO database until the last phenprocoumon or acenocoumarol dispensing in the PHARMO database, the outcome of interest, death, or the end of the study period (31 December 2001), whichever event was earliest. Treatment episodes during follow-up were established for coumarins as well as PIDs. Patients may have had more than one treatment episode of coumarins and PIDs. For each patient, we determined the number of days on coumarins alone and the number of days on coumarins in combination with each individual PID during follow-up. The latter was restricted to the first 28 days of PID use that was started during a coumarin treatment episode as it is likely that, after this period, anticoagulant therapy would be titrated to the use of pharmacokinetically and dynamically interacting PIDs, and the risk of bleeding would return to normal. For PIDs used for less than 28 days, the risk period was defined as the duration of use plus the first 4 days thereafter. A wash-out period of 4 days was taken into account, as the risk of bleeding is not normalized immediately after stopping the PID.

The outcome of interest, i.e., major bleeding, was defined as bleeding requiring hospitalization.

\section{Analysis}

All frequently used PIDs with at least five bleedings, irrespective of the time since start of anticoagulant 
therapy and the number of PIDs involved, were analyzed. However, bleedings may be the result of too high a starting dose of coumarins, and/or a combination of several PIDs may be involved. Therefore, only those bleedings that occurred more than 2 weeks after the start of anticoagulant therapy and that fell within the risk period of a single PID were included in the risk calculation. The incidence rate for major bleeding during use of coumarins alone (i.e., the non-exposed period) was calculated using bleedings on coumarins alone as numerator and person-time on coumarins alone as denominator. Incidence rates for major bleeding during combined use of coumarins and a PID (i.e., the exposed period) were calculated for each PID using bleedings during combined use of coumarins and this PID as numerator and person-time on coumarins in combination with this PID as denominator. The crude relative risk of major bleeding associated with the start of PIDs during anticoagulant therapy was calculated by dividing the incidence rate during the exposed period by that during the non-exposed period. Adjusted estimates of relative risks were computed using a Poisson regression model. Hypertension and history of gastrointestinal bleeding are considered to be risk factors for major bleeding during anticoagulant therapy [11]. Hence, use of antihypertensive drugs during follow-up and history of hospitalization for bleeding or ulcer during the 1-year period before the start of coumarin use were included in the multivariate model along with age and gender. Relative risks were also adjusted for the fact that the exposed and non-exposed periods were measured within the same subjects. To study the effect of involvement of multiple PIDs, adjusted relative risks were also computed including all bleedings.

\section{Results}

The inclusion criteria of this study were met by 19,935 new users of phenprocoumon or acenocoumarol. About two-thirds of the patients were older than 60 years of age (Table 1). The majority of the patients $(95 \%)$ used acenocoumarol, which was mostly prescribed by a medical specialist. We identified 44 PIDs that were started during coumarin use in the first year of follow-up by at least 50 patients. For these 44 PIDs, the association with bleeding was studied.

During follow-up, 552 patients were hospitalized for bleeding. Based on the fact that only PIDs with at least five bleedings were analyzed and the fact that only those bleedings that occurred more than 2 weeks after the start of anticoagulant therapy and that fell within the risk period of a single PID were included in the risk calculation, three groups of patients were distinguished: (1) patients with a bleeding on coumarins alone that occurred more than 2 weeks after start of anticoagulant therapy $(n=189)$, (2) patients with a bleeding on coumarins combined with one of the PIDs with at least five bleedings (doxycycline, amoxicillin/clavulanic acid, sulfamethoxazole/trimethoprim, norfloxacin, diclofenac, ibuprofen, naproxen, omeprazole, antithrombotic salicylates, and tramadol) that occurred more than 2
Table 1 Characteristics of new users of acenocoumarol or phenprocoumon during the period 1992-2000

\begin{tabular}{llll}
\hline Characteristics & & $\begin{array}{l}\text { Number } \\
(n=19,935)\end{array}$ & $\begin{array}{l}\text { Percentage } \\
\text { of total }\end{array}$ \\
\hline Gender & Men & 9,526 & 47.8 \\
Age (years) & Women & 10,409 & 52.2 \\
& $40-50$ & 2,677 & 13.4 \\
& $51-60$ & 3,790 & 19.0 \\
Year of start & $61-70$ & 6,281 & 31.5 \\
& $71-80$ & 7,187 & 36.1 \\
Prescriber at start & $1992-1994$ & 5,429 & 27.2 \\
& $1995-1997$ & 6,578 & 33.0 \\
& $1998-2000$ & 7,928 & 39.8 \\
& General practitioner & 3,114 & 15.6 \\
& Specialist & 16,209 & 81.3 \\
& Cardiologist & 4,386 & 27.1 \\
Type of coumarin at start & Surgeon & 2,874 & 17.7 \\
Number of coumarin treatment & Internist & 2,578 & 15.9 \\
episodes during follow-up & Orthopedist & 2,025 & 12.5 \\
& Other specialists & 4,346 & 26.8 \\
Number of coumarin treatment & Other prescribers & 612 & 3.1 \\
days during follow-up & Acenocoumarol & 18,895 & 94.8 \\
& Phenprocoumon & 1,040 & 5.2 \\
& 1 & 14,884 & 74.7 \\
& 2 & 3,447 & 17.3 \\
& $\geq 3$ & 1,604 & 8.0 \\
& 91-180 & 7,047 & 35.3 \\
& $181-365$ & 3,594 & 18.0 \\
& $366-729$ & 2,282 & 11.5 \\
& $\geq 730$ & 2,550 & 12.8 \\
& & 4,462 & 22.4 \\
& & &
\end{tabular}


weeks after start of anticoagulant therapy $(n=47)$, (3) patients excluded from the analyses, i.e., with a bleeding that occurred during the period that no coumarins were used, that fell outside the risk period, that concerned PIDs with less than five bleedings, that occurred within 2 weeks after start of anticoagulant therapy or that fell within the risk period of multiple PIDs $(n=316)$. Table 2 shows the characteristics of these patients. Table 3 shows the incidence rates and relative risks of major bleeding for the above-mentioned ten PIDs. Antibacterial drugs were associated with a four to seven times increased risk of bleeding, amoxicillin combined with clavulanic acid being a prominent example. Among the non-steroidal anti-inflammatory drugs (NSAIDs), naproxen had the highest relative risk. Antithrombotic salicylates and tramadol were associated with a three times increased risk of bleeding.
By contrast, omeprazole was not associated with a significant increased risk of bleeding. Relative risks did not change when adjusting for the fact that the exposed and non-exposed periods were measured within the same subjects.

When bleedings that occurred within the risk period of more than one PID were included in the analyses, all relative risks largely increased (data not shown). For example, norfloxacin was associated with a 12 times increased risk of bleeding (95\% CI 5.3-26.9) when bleedings in the risk periods of norfloxacin and doxycycline $(n=1)$ or norfloxacin and sulfamethoxazole/trimethoprim $(n=2)$ were included. Tramadol was associated with a 5.5 times increased risk of bleeding (95\% CI 2.3-13.5) when bleedings in the risk periods of tramadol and diclofenac $(n=1)$ or tramadol and naproxen $(n=1)$ were part of the analysis.

Table 2 Characteristics of new users of acenocoumarol or phenprocoumon hospitalized for bleeding during the period 1992-2001

\begin{tabular}{llll}
\hline Characteristics & $\begin{array}{l}\text { Bleedings on } \\
\text { coumarins alone }\end{array}$ & $\begin{array}{c}\text { Bleedings on coumarins } \\
+ \text { potentially interactive drugs }\end{array}$ & $\begin{array}{c}\text { Excluded } \\
\text { bleedings }\end{array}$ \\
\hline $\begin{array}{l}\text { Number of patients } \\
\text { Gender }\end{array}$ & 189 & 47 & 316 \\
$\begin{array}{l}\text { Men } \\
\text { Women }\end{array}$ & $101(53.4 \%)$ & $25(53.2 \%)$ & $165(52.2 \%)$ \\
$\begin{array}{l}\text { Age (years) } \\
40-50\end{array}$ & $88(46.6 \%)$ & $22(46.8 \%)$ & $151(47.8 \%)$ \\
$\begin{array}{l}51-60 \\
61-70\end{array}$ & $20(10.6 \%)$ & $1(2.1 \%)$ & $22(7.0 \%)$ \\
$71-80$ & $25(13.2 \%)$ & $5(10.6 \%)$ & $47(14.9 \%)$ \\
$\begin{array}{l}\text { Use of antihypertensive } \\
\text { drugs during follow-up }\end{array}$ & $57(30.2 \%)$ & $21(44.7 \%)$ & $105(33.2 \%)$ \\
$\begin{array}{l}\text { History of hospitalization } \\
\text { for bleeding or ulcer }\end{array}$ & $87(46.0 \%)$ & $39(83.6 \%)$ & $142(44.9 \%)$ \\
$\begin{array}{l}\text { Exposed and non-exposed } \\
\text { periods during follow-up }\end{array}$ & $152(80.4 \%)$ & $1(2.1 \%)$ & $237(75.0 \%)$ \\
\hline
\end{tabular}

Table 3 Incidence rates and relative risks of bleeding requiring hospitalization associated with the start of potentially interactive drugs during anticoagulant therapy with coumarins. NSAIDs non-steroidal anti-inflammatory drugs, $p y$ patient-years, $I R$ incidence rate, $R R$ relative risk, $C I$ confidence interval

\begin{tabular}{|c|c|c|c|c|c|}
\hline Potentially interactive drug & $\begin{array}{l}\text { Number of } \\
\text { bleedings }^{\mathrm{a}}\end{array}$ & $\begin{array}{l}\text { Number of py } \\
\text { on combined use }\end{array}$ & $\begin{array}{l}\text { IR per } \\
100 \text { py }\end{array}$ & $\begin{array}{l}\operatorname{RR}(95 \% \text { CI }), \\
\text { crude }^{\mathrm{b}}\end{array}$ & $\begin{array}{l}\mathrm{RR}(95 \% \mathrm{CI}) \text {, } \\
\text { adjusted }^{\mathrm{c}}\end{array}$ \\
\hline \multicolumn{6}{|l|}{ Antibacterial drugs } \\
\hline Doxycycline & 6 & 139.6 & 4.3 & $4.2(1.9-9.5)$ & $4.2(1.9-9.5)$ \\
\hline Amoxicillin + clavulanic acid & 6 & 81.5 & 7.4 & $7.2(3.2-16.2)$ & $7.0(3.1-15.8)$ \\
\hline Sulfamethoxazole + trimethoprim & 3 & 44.1 & 6.8 & $6.6(2.1-20.8)$ & $6.2(2.0-19.5)$ \\
\hline Norfloxacin & 3 & 44.8 & 6.7 & $6.5(2.1-20.4)$ & $5.9(1.9-18.6)$ \\
\hline \multicolumn{6}{|l|}{ NSAIDs } \\
\hline Diclofenac & 8 & 295.8 & 2.7 & $2.6(1.3-5.4)$ & $2.6(1.3-5.2)$ \\
\hline Ibuprofen & 5 & 211.2 & 2.4 & $2.3(1.0-5.6)$ & $2.2(0.9-5.4)$ \\
\hline Naproxen & 9 & 131.8 & 6.8 & $6.7(3.4-13.0)$ & $6.5(3.3-12.7)$ \\
\hline Omeprazole & 1 & 171.2 & 0.6 & $0.6(0.1-4.1)$ & $0.6(0.1-4.0)$ \\
\hline Salicylates, antithrombotic & 3 & 103.8 & 2.9 & $2.8(0.9-8.8)$ & $3.0(1.0-9.4)$ \\
\hline Tramadol & 3 & 84.9 & 3.5 & $3.4(1.1-10.8)$ & $3.3(1.1-10.4)$ \\
\hline
\end{tabular}

a Bleedings that fell within the risk period of a single PID and occurred more than 2 weeks after start of anticoagulant therapy ${ }^{\mathrm{b}}$ Calculated by dividing the incidence rate during combined use by the incidence rate during use of coumarins alone (i.e., 189 bleedings on coumarins alone that occurred more than 2 weeks after start of anticoagulant therapy and $6,734,635$ patient-days on coumarins alone $=1.024$ per 100 patient-years $)^{\mathrm{c}}$ Adjusted for age (10-year age classes), gender, history of hospitalization for bleeding or ulcer in the 1-year period before the start of coumarin use, and use of antihypertensive drugs during follow-up 


\section{Discussion}

The results of this population-based cohort study among patients using acenocoumarol or phenprocoumon show that antibacterial drugs, NSAIDs, antithrombotic salicylates, and tramadol, but not omeprazole, are among the main PIDs associated with major bleeding at start of treatment under everyday circumstances. Multiple PIDs seem to have a synergistic adverse effect.

Enhancement of the anticoagulant effect of coumarin anticoagulants, and thus the increased risk of bleeding associated with antibacterial drugs, may result from pharmacokinetic or pharmacodynamic interactions [20]. Pharmacokinetic interactions include inhibition of the metabolism of coumarins or their displacement from plasma proteins. The latter mechanism plays only a minor role compared with other mechanisms. Pharmacodynamic interactions may occur as a result of vitamin $\mathrm{K}$ deficiency that is secondary to elimination of bacterial gut flora or may occur due to direct inhibition of the synthesis of the vitamin K-dependent coagulation factors. Most NSAIDs and antithrombotic salicylates do not influence the intensity of the anticoagulant effect of coumarin anticoagulants, but increase the risk of bleeding by interfering with hemostasis or by their ulcerogenic effect [13]. For tramadol, the presence of an interaction with coumarins is unclear and a possible mechanism for the increased risk of bleeding is unknown [3].

Regarding the combined use of coumarins and PPIs, only omeprazole was co-prescribed with a sufficient frequency to meet the criterion of at least five bleedings for calculation of the relative risk (RR 0.6, Table 2). No bleedings occurred during 5,153 days on coumarins in combination with lansoprazole and only one bleeding occurred during 12,420 days on coumarins in combination with pantoprazole. Extending the analyses for reasons of comparison, pantoprazole, like omeprazole, was not associated with a significant increased risk of bleeding (RR 2.8; 95\% CI 0.4-19.7). Our finding that the results were similar for all PPIs is in line with a recent evaluation of the Food and Drug Administration's database of reported adverse events and drug interactions [10], and disagrees with previous suggestions that omeprazole [19] or omeprazole and lansoprazole [14] bear a higher risk of interactions than pantoprazole.

Some potential limitations should be considered in the interpretation of our study. For omeprazole (and pantoprazole), only one bleeding met the criteria for inclusion in the analyses. This suggests that PPIs did not play a major role in bleeding complications requiring hospitalization during anticoagulant therapy with coumarins, but prohibits judging the relative risks.

In The Netherlands, anticoagulant therapy is monitored at the anticoagulation clinic at a mean interval of 2-3 weeks. Patients are told to inform the anticoagulation clinic about changes in drug use and, if necessary, their intensity of anticoagulation will be measured ear- lier than according to the monitoring scheme. Consequently, bleedings after the start of PIDs may have been prevented by adequate action taken at the anticoagulation clinic, resulting in an underestimation of the relative risks. This does not apply to most NSAIDs and antithrombotic salicylates, as the majority of these drugs are unlikely to influence the intensity of the anticoagulant effect. Dose changes of coumarins and earlier measurement could not be considered in the analyses as this information is not available in the database. Therefore, the results of our study reflect the risk of major bleeding associated with the start of PIDs under everyday circumstances, including reporting by the patient and action taken by the anticoagulation clinic.

The types of coumarin used by our study cohort were acenocoumarol and phenprocoumon. The difference in structure of acenocoumarol and phenprocoumon, although small, may have implications on the relative contribution of CYP enzymes to their metabolism [6]. Therefore, the risk of bleeding associated with the start of inhibitors of CYP1A2, 2C19, and 2C9 possibly differs with the type of anticoagulant used. However, the number of bleedings in users of phenprocoumon was too small to enable studying effect modification. In many countries, warfarin is the coumarin of first choice. The results of our study will largely apply to these countries as well. First, pharmacodynamic interactions with one anticoagulant may well apply to another anticoagulant [18]. Second, PIDs that interact by inhibiting CYP2C9 will affect both acenocoumarol and warfarin [6]. Third, PIDs that do not influence the intensity of the anticoagulant effect of coumarin anticoagulants, but increase the risk of bleeding by interfering with hemostasis or by their ulcerogenic effect, will exert their effect regardless of the type of coumarin used.

Epidemiological studies quantifying the role of drug interactions in bleeding complications in a non-selected population on coumarins under everyday circumstances are scarce. In a Danish population-based cohort study, the incidence of hospitalization for upper gastrointestinal bleeding with use of oral anticoagulants in combination with other drugs was examined [9]. Compared with the general population not exposed to oral anticoagulants, the incidence ratio was $8.0(95 \%$ CI 2.1-20.4) for use of oral anticoagulants combined with non-aspirin NSAIDs. This result is comparable to our study.

In conclusion, in this population-based cohort study among patients using acenocoumarol or phenprocoumon, the main PIDs associated with an increased risk of bleeding were doxycycline, amoxicillin combined with clavulanic acid, sulfamethoxazole combined with trimethoprim, norfloxacin, diclofenac, ibuprofen, naproxen, antithrombotic salicylates, and tramadol. If possible, the use of these drugs should be avoided in patients on coumarins. Regarding antibacterial drugs, more frequent monitoring of anticoagulant therapy may minimize the risk of bleeding complications if there is no therapeutic alternative available. 
Acknowledgements This study was financially supported by AstraZeneca, Germany.

\section{References}

1. Caron J, Libersa C, Thomas C (1996) Drugs affecting blood clotting, fibrinolysis, and hemostasis. In: Dukes MNG (ed) Meyler's side effects of drugs: an encyclopedia of adverse reactions and interactions, 13th edn. Elsevier Science, Amsterdam, pp 1008-1055

2. Catalan VS, LeLorier J (2000) Predictors of long-term persistence on statins in a subsidized clinical population. Value Health 3(6):417-426

3. Commissie Interacterende medicatie cumarines (2002) Standaard afhandeling cumarine-interacties [cited 2003 Feb 12]. Available from: URL: http://www.fnt.nl/artsen/index.html

4. Harder S, Thurmann P (1996) Clinically important drug interactions with anticoagulants. An update. Clin Pharmacokinet 30(6):416-444

5. Herings R (1993) PHARMO: a record linkage system for postmarketing surveillance of prescription drugs in The Netherlands [dissertation]. Utrecht University, Department of Pharmaco-epidemiology and -therapy, Utrecht

6. Hermans JJ, Thijssen HH (1993) Human liver microsomal metabolism of the enantiomers of warfarin and acenocoumarol: P450 isozyme diversity determines the differences in their pharmacokinetics. Br J Pharmacol 110(1): 482-490

7. Holbrook AM, Wells PS, Crowther NR (1996) Pharmacokinetics and drug interactions with warfarin. In: Poller L, Hirsch J (eds) Oral anticoagulants. Arnold, London, pp 30-48

8. Indiana University Department of Medicine (2003) Cytochrome P450 Drug Interaction Table [cited 2003 Feb 12]. Available from: URL: http://medicine.iupui.edu/flockhart

9. Johnsen SP, Sorensen HT, Mellemkjoer L, Blot WJ, Nielsen GL, McLaughlin JK et al (2001) Hospitalisation for upper gastrointestinal bleeding associated with use of oral anticoagulants. Thromb Haemost 86(2):563-568

10. Labenz J, Petersen KU, Rosch W, Koelz HR (2003) A summary of food and drug administration-reported adverse events and drug interactions occurring during therapy with omeprazole, lansoprazole and pantoprazole. Aliment Pharmacol Ther 17(8):1015-1019

11. Landefeld CS, Beyth RJ (1993) Anticoagulant-related bleeding: clinical epidemiology, prediction, and prevention. Am J Med 95(3):315-328
12. Li X-Q, Andersson TB, Ahlström M, Weidolf L (2004) Comparison of inhibitory effects of the proton pump inhibiting drugs omeprazole, esomeprazole, lansoprazole, pantoprazole and rabeprazole on human cytochrome P450 activities. Drug Metab Dispos 32(8):821-827

13. Majerus PW, Tollefsen DM (2001) Anticoagulant, thrombolytic, and antiplatelet drugs. In: Hardman JG, Goodman Gilman A, Limbird LE (eds) The pharmacological basis of therapeutics, 10th edn. McGraw-Hill, New York

14. McCarthy DM, McLaughlin TP, Griffis DL, Yazdani C (2003) Impact of cotherapy with some proton pump inhibitors on medical claims among HMO patients already using other common drugs also cleared by cytochrome P450. Am J Ther 10(5):330-340

15. van der Meer FJ, Rosendaal FR, Vandenbroucke JP, Briet E (1996) Assessment of a bleeding risk index in two cohorts of patients treated with oral anticoagulants. Thromb Haemost 76(1):12-16

16. Palareti G, Leali N, Coccheri S, Poggi M, Manotti C, D‘Angelo A et al (1996) Bleeding complications of oral anticoagulant treatment: an inception-cohort, prospective collaborative study (ISCOAT). Italian Study on Complications of Oral Anticoagulant Therapy. Lancet 348(9025):423-428

17. Penning-van Beest FJA, van Meegen E, Rosendaal FR, Stricker BHCh (2001) Drug interactions as a cause of overanticoagulation on phenprocoumon or acenocoumarol predominantly concern antibacterial drugs. Clin Pharmacol Ther 69(6):451-457

18. Reynolds JEF (ed) (1999) Anticoagulants. Martindale: the extra pharmacopoeia, 32nd edn. The Pharmaceutical Press, London

19. Steinijans VW, Huber R, Hartmann M, Zech K, Bliesath H, Wurst W et al (1996) Lack of pantoprazole drug interactions in man: an updated review. Int J Clin Pharmacol Ther 34(Suppl 1):S31-S50

20. Stockley IH (1996) Drug interactions, 4th edn. The Pharmaceutical Press, London

21. Visser LE, Penning-van Beest FJA, Kasbergen AA, De Smet PA, Vulto AG, Hofman A et al (2002) Overanticoagulation associated with combined use of antifungal agents and coumarin anticoagulants. Clin Pharmacol Ther 71(6):496-502

22. Visser LE, Penning-van Beest FJA, Kasbergen AA, De Smet PA, Vulto AG, Hofman A et al (2002) Overanticoagulation associated with combined use of antibacterial drugs and acenocoumarol or phenprocoumon anticoagulants. Thromb Haemost 88(5):705-710

23. Visser LE, Penning-van Beest FJA, Wilson JH, Vulto AG, Kasbergen AA, De Smet PA et al (2004) Overanticoagulation associated with combined use of lactulose and coumarin anticoagulants. Br J Clin Pharmacol 57(4):522-524 